\title{
Comparison of Sliding Mode Control and Fuzzy Logic control applied to Variable Speed Wind Energy Conversion Systems
}

\author{
Zine Souhila ${ }^{1}$, Mazari Benyounes ${ }^{1}$, Bouzid Mohamed Amine ${ }^{2}$ \\ ${ }^{1}$ Laboratory of development of electrical drives (LDEE) \\ University of Sciences and Technology \\ Oran, Algeria \\ souracha2005@yahoo.fr \\ 2 Laboratory of Intelligent Control and Electrical \\ Power Systems \\ University Djilali LIABES \\ Sidi Bel Abbes, Algeria
}

\begin{abstract}
Wind energy features prominently as a supplementary energy booster does not pollute and is inexhaustible. However, its high cost is a major constraint especially on the less windy sites. The purpose of wind energy systems is to maximize energy efficiency and to extract maximum power from the wind speed. In this case,the MPPT control becomes important. To realize this control, strategy conventional Proportional and Integral $(\mathrm{PI})$ controller is usually used. However, this strategy cannot achieve better performance. This pape proposes other control methods of a turbine, which optimize its production, such as fuzzy logic, sliding mode control. These methods improve the quality and energy efficiency. The proposed Sliding Mode Control (SMC) strategy and the fuzzy controllers have presented attractive features, such as robustness to parametric uncertainties of the turbine, simplicity of its design and good performances. The simulation result under MatlablSimulink has validated the performance of the proposed MPPT strategies.
\end{abstract}

Keywords- Heat transfer enhancement factor, Ceramic Plate-Fin (PFHE) Heat Exchanger, Nusselt number, Schmidt number, pressure drop Wind turbine, maximum power point tracking; Sliding Mode Control; fuzzy logic control.

\section{INTRODUCTION}

In recent years, the strong industrialization and the increase in household appliances led to huge power needs. To face this growing demand and to avoid pollution fossil fuels (oil, gas), several countries were interested in renewables energies. In this context, wind energy occupies an important place as a supplementary energy booster. It does not pollute and it is inexhaustible. For this, improved control strategies to obtain maximum performance and longevity of its life become increasingly necessary. [1]

Several researches on wind control were conducted. The latest generation of wind turbines operates at variable speed, which offers a higher yield of energy compared to fixed-speed turbines [2\%]. The rotational speed of turbine varies with changing wind speed to maintain the operating point of maximum efficiency in order to get the coefficient of maximum power and the maximum power point tracking. In this case, the MPPT control becomes important [3]. The purpose of wind energy systems is to maximize energy efficiency and extract maximum power from the wind speed; this can be achieved through different methods. Optimum power/torque tracking is the most popular control strategy. To realize this control strategy, conventional Proportional and Integral controller is usually used [4]. However, this strategy cannot achieve better performance. To solve this problem, there are other control methods such as fuzzy logic and sliding mode control.

The sliding mode control is a variable structure control, that it's a non-linear control [5]. Proposed in the early 1950s, its success has been proven in control problems, since it is able to tackle system uncertainties and external disturbances with good robustness. This control is characterized by some advantages such as: high precision, rapid dynamic response, stability, simplicity of its design and its implementation. Its major drawback is the presence of chattering. [6]

The fuzzy controllers have presented encouraging performance in the treatment of nonlinear systems because they do not require a wellknown system. The fuzzy controller is has interesting features, such as simplicity, good performance, and automation [7]. However, its major drawback lies in the hardware and software implementation due to the high computational load. [8]

The main objective of this paper is to propose a robust control to achieve better performance. This paper is organized as follows: Section II describes Wind Energy Conversion System (WECS); Section III develops control strategies. Sliding mode control and fuzzy logic control are designed. In section IV, simulation results show the performance of the proposed approach. Conclusion is presented in section V. The proposed strategy is compared with conventional PI controllers and confirmed superiorly in MATLAB / Simulink environment. 


\section{WECS SYSTEM MODELING}

The Wind Energy Conversion System (WECS) is shown in Fig1. It consists of a turbine, a rectifier, a PMSG and an inverter connected to the grid.

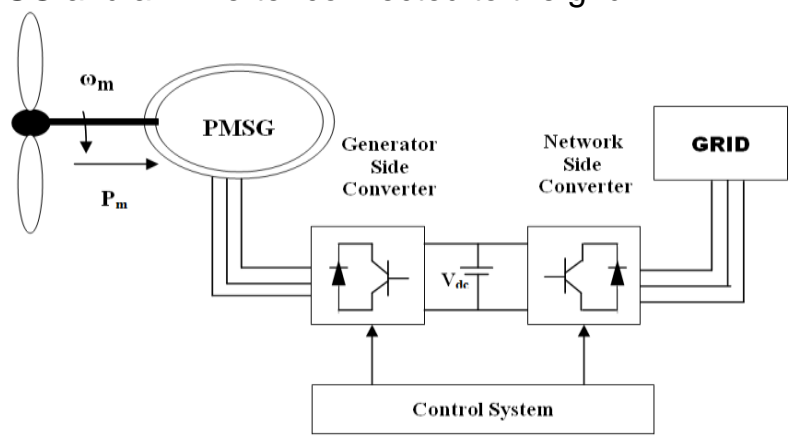

Fig. 1. The structure of wind system based on permanent magnet synchronous machine

Wind turbines are designed to produce electrical energy as cheaply as possible and give maximum power at any speed of the wind. All wind turbines are therefore designed with some sort of power control. This standard control law keeps the turbine operating at the peak of its Cp curve [9]. However, this control has an important problem since wind speed fluctuations force the turbine to operate off the peak of its $\mathrm{Cp}$ curve most of the time, resulting in less energy capture [9]. Therefore, our work will be based on the mechanical part of the chain (turbine), in order to propose a robust control to achieve better performance.

\section{A. Modeling of wind turbine}

The mathematical relation for the mechanical turbine power can be expressed as follows [10]:

$$
P_{m}=C_{p}(\lambda, \beta) P_{w}=C_{p}(\lambda, \beta) \frac{\rho A V^{s}}{2}
$$

Where $P w$ is the extracted power from the wind; $\rho$ is the air density $[\mathrm{kg} / \mathrm{m} 3] ; V_{w}$ is the wind speed; $A$ is the area swept by the rotor blades of the wind turbine and $C_{p}$ is the power coefficient which is a function of both blade pitch angle $\beta$ and tip speed ratio $\lambda$. [11]:

Tip speed ratio as given in the following equation

$$
\lambda=\frac{R \omega_{m}}{V_{w e}}
$$

Where $\omega_{\mathrm{m}}$ is the angular speed of the turbine rotor and $\mathrm{R}$ is the radius of the turbine blades. [12]:

The power coefficient $\mathrm{Cp}$ can be expressed as

$$
C_{p}(\lambda, \beta)=C_{1}\left(C_{2} \frac{1}{\lambda}-C_{3} \beta-C_{4}\right) \exp ^{\left(-C_{5 \frac{1}{\lambda}}\right)}+C_{6} \lambda
$$

Where

$\frac{1}{\lambda_{i}}=\frac{1}{\lambda+0.08 \beta}-\left(\frac{0.035}{\beta^{3}+1}\right)$

$\mathrm{C} 1=0.5176, \mathrm{C} 2=116, \mathrm{C} 3=0.4, \mathrm{C} 4=5, \mathrm{C} 5=21$ and $\mathrm{C} 6=0.0068 . \Omega$

Fig 2 indicates the relation between $C_{p}$ and $\lambda$ for different pitch angles.

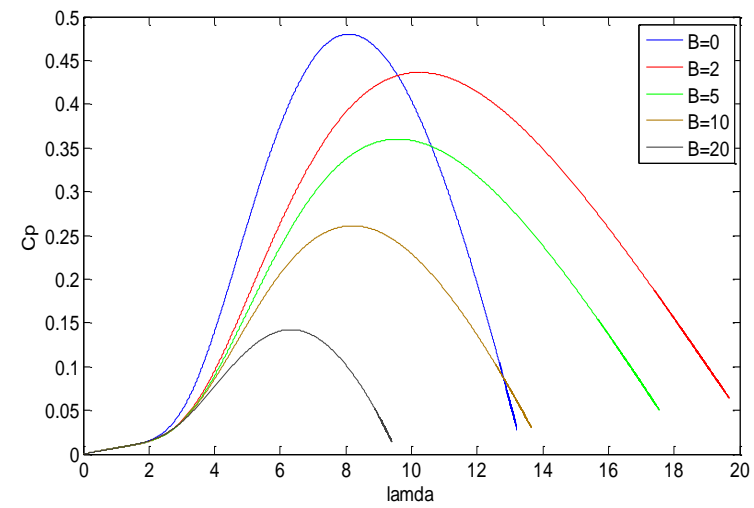

Fig. 2. Characteristics of the power coefficient as function of $\lambda$ and $\beta$

It can be noticed that when pitch-angle are fixed at a value of 0 , we get the nominal value of $C_{p}$ about 0.48 for $\lambda$ of 8.1 . So the maximum power can be obtained from the wind.

The torque of the wind turbine can be expressed as:

$$
T=C_{p}(\lambda, \beta) \frac{\rho A V^{\mathrm{s}}}{2 \lambda}
$$

The mechanical equation is given by:

$$
\begin{aligned}
& I \frac{d \omega_{m}}{d t}=T-T_{e}-B W_{m} \\
& \text { III. MAXIMUM POWER POINT TRACKING } \\
& \text { CONTROL }
\end{aligned}
$$

\section{MAXIMUM POWER POINT TRACKING}

The MPPT control is divided into three parts:

- The first part concerns the realization of the classical MPPT; this strategy can be realized using conventional Proportional and Integral (PI) controller, such as in Fig 3.

- In the second part, we will replace conventional Proportional and Integral (PI) controller by sliding mode control, such as in Fig 4 .

- In the third part, conventional Proportional and Integral (PI) controller is replaced by fuzzy logic controller.

\section{A. Maximum Power Point Tracking (MPPT) Principle}

To extract the maximum power, we proceed to control the speed of the wind turbine. The wind speed measured using an anemometer is used as the reference for the speed control loop. The Maximum Power Point Tacking (MPPT) algorithm gives the reference torque applied to the turbine, so that extracted power is maximum at any wind velocity below the maximum value [13], as shown in Fig 3. 


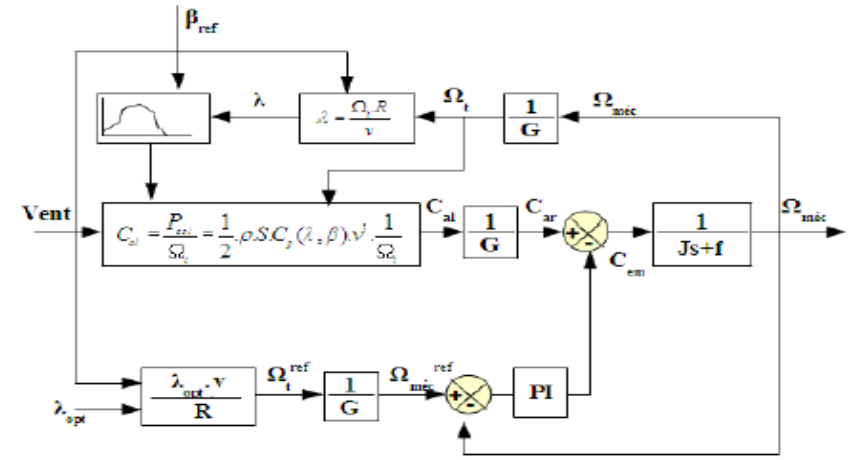

Fig. 3. Bloc diagram of torque control scheme

To ensure maximum efficiency of the turbine, it necessary that power coefficient remains optimum. The use of the linearization approach is common in addressing the control problem of wind turbines. However, due to the inevitable uncertainties inherent in the system, this strategy cannot achieve better performance. To solve this problem, we will use sliding mode control, which takes into account these control problems. [9]

\section{B. Sliding control}

The sliding mode is a special mode of operation of the variable structure system. It was a great success in recent years; this is due to the simplicity of its implementation and robustness, compared with the uncertainties of system and external disturbances, vitiating the process. [5]

Control by sliding mode is divided into three parts. The first step is to select a sliding surface, which models the desired closed-loop performance in the state variable space. Then, we impose the condition of convergence, and then the command must be designed so that the system state trajectories are directed towards the sliding surface and stay on it. [14]

\section{1) Choice of the sliding surface}

A non-linear system is presented by the following form:

$$
\dot{\mathrm{X}}=f(\mathrm{X}, \mathrm{t})+\mathrm{g}(\mathrm{X}, \mathrm{t}) \mathrm{u}(\mathrm{X}, \mathrm{t})
$$

Where $f(\mathrm{X}, \mathrm{t}), \mathrm{g}(\mathrm{X}, \mathrm{t})$ are both non-linear functions

Taking JJ Slotine equation, to determine the sliding surface, which is given by [14]:

$$
\begin{gathered}
S(X)=\left(\frac{d}{d t}+\lambda\right)^{n-1} e \\
e=X^{d}-X
\end{gathered}
$$

e: error on the controlled variable;

$-\lambda$ : positive coefficient;

$-n$ : order of the system;

$-X^{\mathrm{d}}$ : desired size;

-X: state of the controlled variable size.
So that the surface is attractive and invariant, regulator sliding mode should be chosen to satisfy the Lyapunov stability criterion.

\section{2) Convergence condition}

The convergence condition is defined by the following equation:

$S(X) \dot{S}(X) \leq 0$

3) Control

The control structure is defined by the following equation:

$\mathrm{U}=\mathrm{U}_{\mathrm{eq}}+\mathrm{U}_{\mathrm{n}}$

With $U_{\text {eq }}$-the component of the equivalent control, $\mathrm{U}_{\mathrm{n}}$-component of discontinuous control

$\mathrm{U}_{\mathrm{n}}=\mathrm{U}_{\max } \operatorname{sgn}(\mathrm{S}(\mathrm{X}))$

Where:

$-\operatorname{sgn}(\mathrm{S}(\mathrm{X}))$ : sign function,

- $U_{\max }$ : positive gain.

\section{4) Speed control}

To control the speed, the degree of the sliding surface is taken equal to 1 ; the sliding surface $S(w)$ is:

$\mathrm{S}(\mathrm{w})=\mathrm{w}_{m \mathrm{ref}}-\mathrm{w}_{m}$

By deriving equation (12), we obtain:

$\dot{S}(\mathrm{w})=\dot{\mathrm{W}}_{\text {ref }}-\dot{\mathrm{w}}$

By replacing the expression of speed, we get:

$\dot{\mathrm{S}}(\mathrm{w})=\dot{\mathrm{W}}_{\text {ref }} \frac{\mathrm{T}-\mathrm{Te}}{J}+\frac{F}{J} \dot{\mathrm{w}}$

During the sliding mode surface $S(w)=\dot{S}(w)=0$. $\dot{\mathrm{W}}_{\text {ref }}-\frac{\mathrm{T}-\mathrm{Te}}{I}+\frac{F}{J} \dot{\mathrm{w}}=0$

Then we obtain

$\left\{\begin{array}{l}U_{\text {eq }}=T-J \dot{\mathrm{w}}_{r e f}-F \dot{\mathrm{w}} \\ U_{m}=U_{\operatorname{mnx}} \operatorname{sgn}(S(\mathrm{w}))\end{array}\right.$

The proposed control scheme is shown in Fig 4

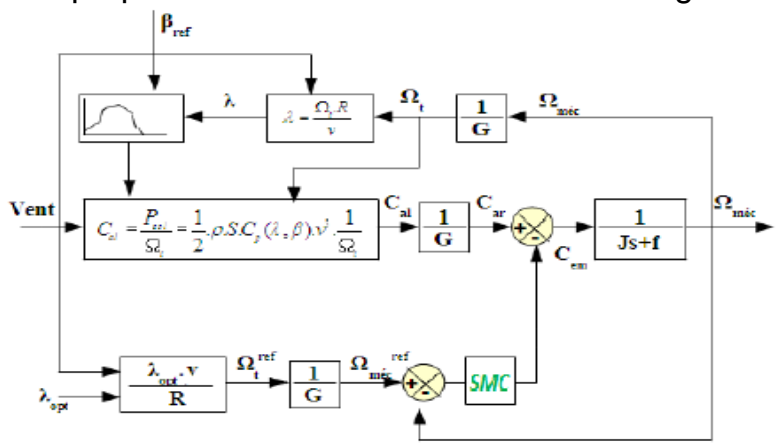

Fig. 4. The proposed strategy scheme 


\section{Fuzzy Logic Control}

Fuzzy logic controllers are similar to how human beings make decisions. The fuzzy control is used where the nonlinearities are significant, as in the case of wind. The fuzzy control did not need to know dynamics of the system [15].

A fuzzy controller generally consists of four subsystems, in which two parts have duty of transformation [16]:

Fuzzifier (first transformation),

Fuzzy rule base,

Inference engine,

And defuzzifier (second transformation).

In a control system, the fuzzy system operates in real time to find the values optimal or near of optimum. The use of fuzzy rules base ensures that the operating the device remains within the desired range of operation [17].

\section{1) Fuzzy logic controller}

Fig. 5 shows the general structure of the control system fuzzy logic used in our work. Control output $u$ is deduced from the error (e) and change of error $(\Delta \mathrm{e})$. Control rules are designed to assign to a fuzzy set of the control output $u$ for each combination of fuzzy sets of e and $(\Delta e)$.

Fig. 5. Structure of a fuzzy control system

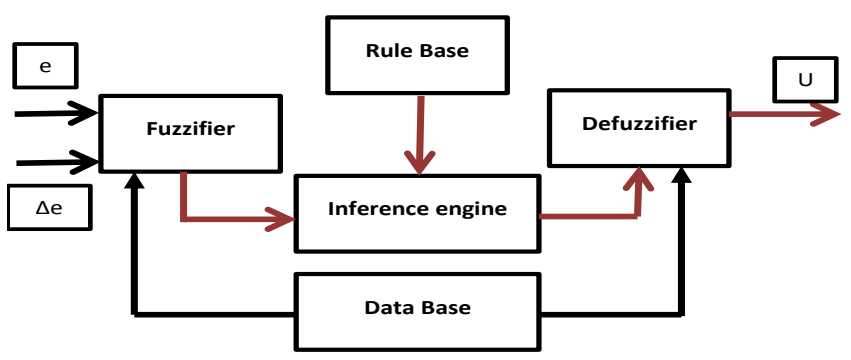

The rules of $\mathrm{Kp}$ and $\mathrm{Ki}$ are presented in the Table 1 and Table 2. Error is represented by the lines and error change $\Delta e$, by columns. Each interaction of $e$ and $\Delta$ e determines the level of output corresponding to $u$. [18]

Table 1 . Rule base of $\mathrm{Kp}$

\begin{tabular}{|c|c|c|c|c|c|c|}
\hline \multirow{2}{*}{$\mathbf{u}$} & \multicolumn{5}{|c|}{$\Delta \mathbf{e}$} \\
\cline { 3 - 7 } \multicolumn{1}{|c|}{} & NB & NS & ZR & PS & PB \\
\hline \multirow{4}{*}{ e } & NB & VB & VB & VB & VB & VB \\
\cline { 2 - 7 } & NS & B & B & B & BM & VB \\
\cline { 2 - 7 } & ZR & ZR & ZR & NM & S & S \\
\cline { 2 - 7 } & PS & B & B & B & BM & VB \\
\cline { 2 - 7 } & PB & VB & VB & VB & VB & VB \\
\hline
\end{tabular}

Table 2. Rule base of $\mathrm{Ki}$

\begin{tabular}{|c|c|c|c|c|c|c|}
\hline \multicolumn{2}{|c|}{} & \multicolumn{5}{c|}{$\Delta \mathbf{c}$} \\
\cline { 3 - 7 } & & NB & NS & ZR & PS & PB \\
\hline \multirow{4}{*}{ e } & NB & M & M & M & M & M \\
\cline { 2 - 7 } & NS & S & S & S & S & S \\
\cline { 2 - 7 } & ZR & NM & NM & ZR & NM & NM \\
\cline { 2 - 7 } & PS & S & S & S & S & S \\
\cline { 2 - 8 } & PB & M & M & M & M & M \\
\cline { 2 - 7 } & & &
\end{tabular}

The abbreviations of Table 1 are defined as follows: VB is very big; NB is Negative Big; NM is Negative Medium; NS is Negative Small; ZR is Zero; PS is Positive Small; BM is Positive Medium; PB is Positive Big; $M$ is medium; $B$ is Big and $S$ is small. These abbreviations are labels of fuzzy sets and their corresponding membership functions are depicted in Figures 6-8.

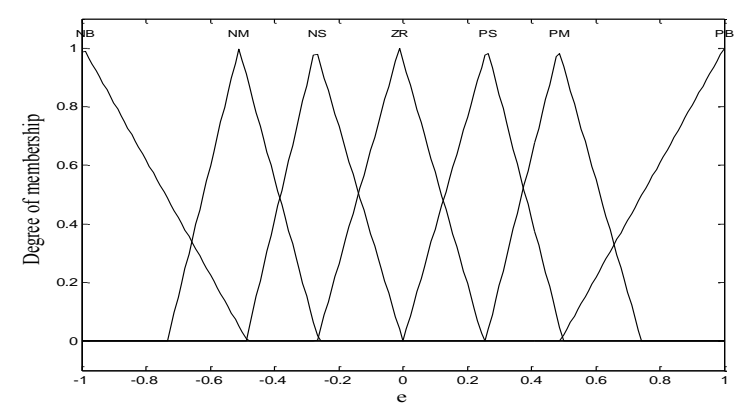

Fig. 6. Membership function for input e

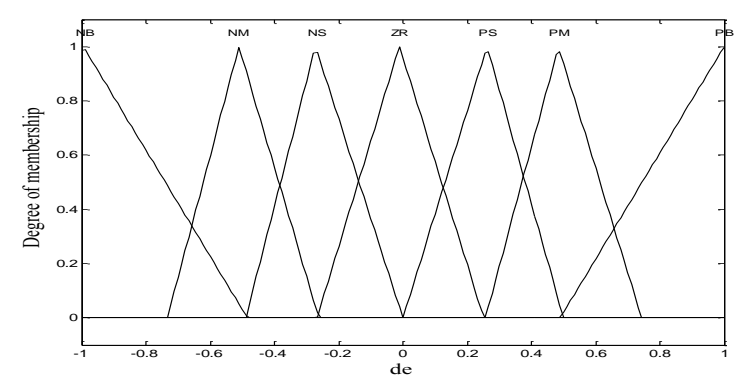

Fig. 7. Membership functions for input $\Delta \mathrm{e}$

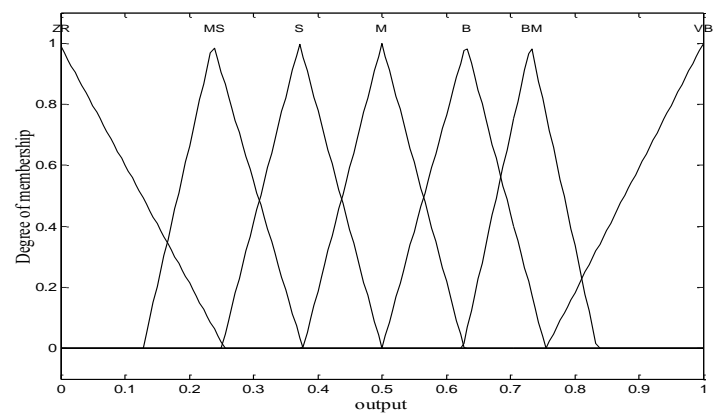

Fig. 8. Membership function of output 
In this paper, the triangular membership function, the max-min reasoning method, and the center of gravity defuzzification method are used. The parameters of the PI speed controller are adjusted by using two fuzzy logic controllers to define $\mathrm{Kp}$ and Ki.

\section{SIMULATION RESULT}

A global simulation of the system under Matlab/Simulink is realized. Table 1 illustrates system's parameters. Fig 9 shows the wind speed.

Table 3. Simulation parameters

\begin{tabular}{|l|l|l|}
\hline \multicolumn{3}{|l|}{ Wind Turbine Parameters } \\
\hline $\mathrm{R}=3 \mathrm{~m}$ & $\rho=1.22$ & $\mathrm{~J}=0.042 \mathrm{Kg} \cdot \mathrm{m} 2$ \\
\hline $\mathrm{C} p_{\text {opt }}=0.48$ & $\lambda \mathrm{opt}=8.1$ & $\begin{array}{l}B=0.017 \text { N.m.s- } \\
1\end{array}$ \\
\hline
\end{tabular}

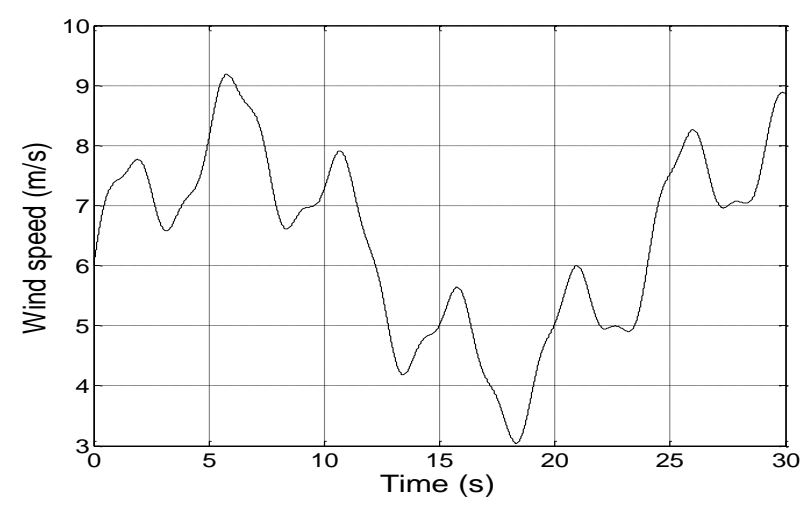

Fig. 9. Variation of wind speed $(\mathrm{m} / \mathrm{s})$

The power coefficient variations are shown in Fig 10 . It can be clearly seen that the Sliding Mode Controller provides better than the PI and Fuzzy Logic controllers. The uses of the sliding mode greatly reduces the fluctuation, which ensures optimization of the extracted power.

Figure 11 shows the efficiency of the Sliding Mode Control to maintain the speed ratio at its optimum value under varying wind conditions. The $\mathrm{PI}$ and Fuzzy Logic controllers stay oscillating around optimal value.

The average output power as a function of wind speed is shown in Fig 12; this power is the maximum extracted power from available wind power, keeping the value of the power coefficient at its optimum value. One notices the sliding mode controller and fuzzy controller show better performances than $\mathrm{Pl}$ controller in optimizing the power conversion performance.

The speed curves of Fig 13 illustrate the high performance of the three developed controllers, and allow having high dynamic responses in tracking the desired speed reference, especially with the use of the sliding mode control.

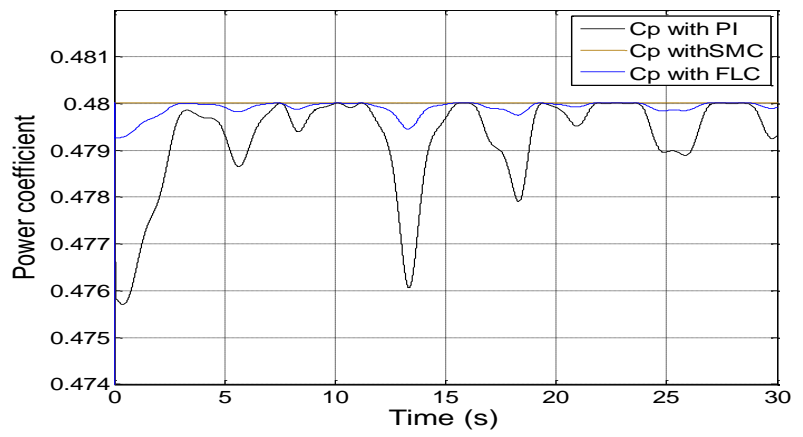

Fig.10. Power coefficient

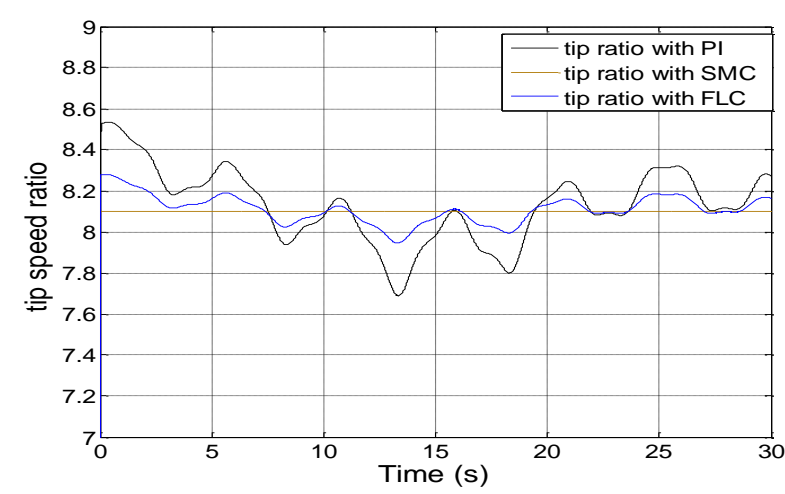

Fig. 11. Variation of tip speed ratio

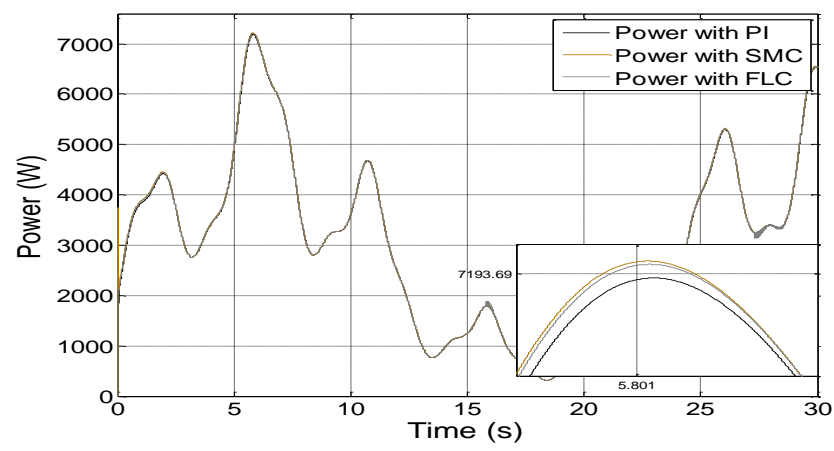

Fig. 12. Turbine Power

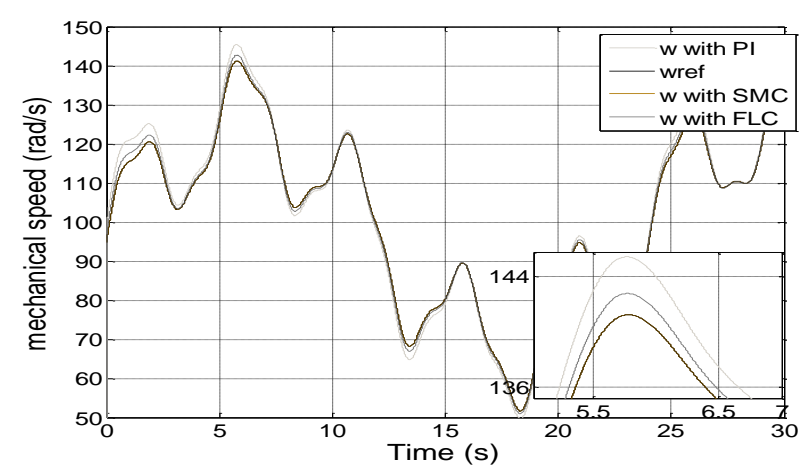

Fig.13. Speed of turbine 
This paper adresses the problem of controlling power generation in variable speed wind turbines. A torque controller has been developed to follow a speed trajectory that allows the wind turbine to operate with maximum power extraction in below rated wind speed.

For this purpose, three control strategies have been proposed. The first uses a conventional proportional and integral $(\mathrm{PI})$ controller, the second uses the sliding mode control, whereas the third uses Fuzzy logic controller.

Interesting features, such as high precision, rapid dynamic response, stability, simplicity of its design and its implementation, characterize sliding mode control and fuzzy controllers.

The simulation result shows that Sliding Mode Controller has better performances than a conventional Proportional and Integral controller, since the coefficient is maintained at its optimum value, thereby ensuring the optimization of the extracted power.

\section{REFERENCES}

[1] Ghennam, T.« Supervison d'une ferme éolienne pour son intergation dans la gestion d'un réseau électrique, Apports des convertisseurs multi niveaux au réglages des éoliennes à base de machine asynchrone à double alimentation », Ecole militaire polyctechnique d'Alger. 2011.

[2] Beltran, B. «Maximisation de la puissance produite par une génératrice asynchrone Double alimentation d'une éolienne par mode glissant d'ordre supérieur ». JCGe. 16-17 December 2008.

[3] Hussein, M. H., Orabi, M., Ahmed, M. E., Abd ElWahab, M. A. and Hamada, M. M. "Simple Direct Sensorless Control of Permanent Magnet Synchronous Generator Wind Turbine ». Proceedings of the 14th international Middle East Power Systems Conference (MEPCON'10). Cairo University, Egypt. 19-December2010.

[4] Haque, M. E. , Muttaqi, K. M. and Negnevitsky, M. « Control of a standalone variable speed wind turbine with a permanent magnet synchronous generator ", in 2008 IEEE Power and Energy Society General Meeting - Conversion and Delivery of Electrical Energy in the 21st Century. 2008. P. 109.

[5] Aissaoui, A. G., ABID, H., ABID, M. and TAHOUR, A. "Commande par la logique floue et mode Glissant d'une machine synchrone autopilotée ", Rev Roum SciTechn-Électrotechn Énerg, vol. 52, no 1, p. 89104.

[6] Zhang, L., Chunliang, E., Li, H. and Xu, H. «A new pitch control strategy for wind turbines base on quasisliding mode control ». In Sustainable Power Generation and Supply 2009 SUPERGEN'09 International Conference. Pp. 1-4.
[7] Gargoom, A., Haruni, A. M. O, Haque, M. E. and Negnevitsky, M. « Voltage and frequency stabilizer based on fuzzy logic control for three-level NPC converters in stand-alone wind energy systems $» . \mathrm{In}$ Power and Energy Society General Meeting, 2010 IEEE, 2010, p. 1-7.

[8] Y. Mihoub, D. Toumi, B. Mazari, et S. Hassaine, "Design and Implementation of an Adaptive PI Fuzzy Controller to Improve the Speed Control of Induction Motor. », Int. Rev. Electr. Eng., vol. 5, no 2, 2010.

[9] B. Beltran, T. Ahmed-Ali, et M. E. H. Benbouzid, "Sliding mode power control of variable-speed wind energy conversion systems », Energy Convers. IEEE Trans. On, vol. 23, no 2, p. 551-558, 2008.

[10] Chih-Hong Lin, "Recurrent modified Elman neural network control of PM synchronous generator system using wind turbine emulator of PM synchronous servo motor drive", International Journal of Electrical Power \& Energy Systems, Volume 52, November 2013, Pages 143-160

[11] S.M. Muyeen, Ahmed Al-Durra, J. Tamura, Variable speed wind turbine generator system with current controlled voltage source inverter, Energy Conversion and Management, Volume52, Issue 7, July 2011, Pages 2688-2694

[12] Robert Gasch, JochenTwele. Wind Power Plants: Fundamentals, Design, Construction and Operation, Springer 2012

[13] M. E. Emna, K. Adel, et M. F. Mimouni, «The Wind Energy Conversion System Using PMSG Controlled by Vector Control and SMC Strategies ", Int. J. Renew. EnergyRes. IJRER, vol. 3, no 1, p. 4150, 2013.

[14] Jiabing Hu; Heng Nian; Bin Hu; Yikang He; Zhu, Z.Q., "Direct Active and Reactive Power Regulation of DFIG Using Sliding-Mode Control Approach," Energy Conversion, IEEE Transactions on , vol.25, no.4, pp.1028,1039, Dec. 2010

[15] J. Zhang, M. Cheng, Z. Chen, et X. Fu, « Pitch angle control for variable speed wind turbines », in Electric Utility Deregulation and Restructuring and Power Technologies, 2008. DRPT 2008. Third International Conference on, 2008, p. 2691-2696.

[16] A. Rahideh, M. Karimi, A. Shakeri, et M. Azadi, " High performance direct torque control of a PMSM using fuzzy logic and genetic algorithm », in Electric Machines \& Drives Conference, 2007. IEMDC'07. IEEE International, 2007, vol. 2, p. 932-937.

[17] A. G. Aissaoui, A. Tahour, N. Essounbouli, F. Nollet, M. Abid, et M. I. Chergui, «A Fuzzy-PI control to extract an optimal power from wind turbine », Energy Convers. Manag., vol. 65, p. 688-696, 2013.

[18] A. V. A. Macedo et W. S. Mota, «Wind turbine pitch angle control using Fuzzy Logic », in Transmission and Distribution: Latin America Conference and Exposition (T D-LA), 2012 Sixth IEEE/PES, 2012, p. 16. 